\title{
Measurement of fasting breath hydrogen concentration as a simple diagnostic method for pancreatic exocrine insufficiency
}

\author{
Kota Uetsuki ${ }^{1}$, Hiroki Kawashima ${ }^{2} \mathbb{0}$, Eizaburo Ohno ${ }^{1}$, Takuya Ishikawa ${ }^{1}$, Tadashi lida' ${ }^{1}$, Kenta Yamamoto ${ }^{1}$, \\ Kazuhiro Furukawa', Masanao Nakamura' ${ }^{1}$ Takashi Honda ${ }^{1}$, Masatoshi Ishigami ${ }^{1}$, Yoshiki Hirooka ${ }^{3}$ and \\ Mitsuhiro Fujishiro'
}

\begin{abstract}
Background: Pancreatic exocrine insufficiency (PEI) is associated with the outcome of pancreatic disease. However, there is no method for assessing PEl that can be used noninvasively and easily for outpatient. It has been reported that changes in intestinal bacteria caused by PEI may increase breath hydrogen concentration (BHC) levels during glucose or lactose loading. We have evaluated the usefulness of fasting breath hydrogen concentration (FBHC) measurement without glucose loading for the evaluation of PEl.
\end{abstract}

Methods: Sixty patients underwent FBHC measurement, BT-PABA testing, and microbiome analysis. They were classified into PEl group (PABA excretion rate $<73.4 \%, n=30)$ and non-PEl group $(n=30)$. The FBHC of the two groups were compared, and the diagnostic ability of PEl by them was evaluated. The $16 \mathrm{~s} r R N A$ (V3-V4) from fecal samples was analyzed by MiSeq.

Results: FBHC levels was higher in the PEl group 15.70 (1.4 to 77.0) ppm than in the non-PEI group 2.80 (0.7 to 28.2) $\mathrm{ppm}(P<0.0001)$. FBHC was negatively correlated with PABA excretion rate $(r=-0.523, P<0.001)$. The cutoff value of FBHC of 10.7 ppm ( $95 \% \mathrm{Cl}: 0.678-0.913, P<0.001)$ showed a sensitivity of $73.3 \%$ and specificity of $83.3 \%$ for PEl diagnosis. In the PEl group, there was a significant increase of relative abundance of phylum Firmicutes $(P<0.05)$ and the genus Clostridium $(P<0.05)$.

Conclusion: $\mathrm{FBHC}$ shows good potential as a simple and repeatable test for the diagnosis of PEI. The elevated FBHC levels may be caused by hydrogen-producing bacteria such as Clostridium.

Keywords: Pancreatic exocrine insufficiency, Fasting breath hydrogen concentration, Pancreatic disease, Noninvasive measurement

\section{Background}

Pancreatic exocrine insufficiency (PEI) is defined as 'a state of declined pancreatic enzyme activity in the intestinal lumen to a level below the threshold required to

\footnotetext{
*Correspondence: h-kawa@med.nagoya-u.ac.jp

${ }^{2}$ Department of Endoscopy, Nagoya University Hospital, 65 Tsuruma-cho,

Showa-ku, Nagoya 466-8550, Japan

Full list of author information is available at the end of the article
}

maintain normal digestion' [1]. In pancreatic disease, PEI occurs at a high frequency and causes indigestion and nutritional deficiency, which have a negative influence on nutritional status and quality of life (QOL) [2]. Pancreatic enzyme replacement therapy (PERT) significantly improves overall survival time, treatment tolerability, symptoms, and QOL of patients with pancreatic diseases such as pancreatic carcinoma (PC) and chronic pancreatitis $(\mathrm{CP})[3,4]$. This shows the importance of appropriate 
diagnosis of PEI and introduction of treatment. There are many diagnostic methods for PEI, but no consensus on the best approach in clinical practice [5]. The BT-PABA test, which is the only method covered by national health insurance in Japan, is a complicated process that requires about a 6-h test time and fasting.

The breath hydrogen test (BHT) for pancreatic disease was first examined in the 1960s, and this test is now mainly utilized as an indirect diagnostic method for small intestinal bacterial overgrowth syndrome (SIBO), which complicates some CP cases [6]. A study in a small number of patients suggested a relationship between PEI and SIBO [7], but the test requires measurement of breath hydrogen continuously for about $2-4 \mathrm{~h}$ in a fasting state after glucose loading $[8,9]$. Also, patients with a high fasting breath hydrogen concentration (FBHC) were excluded in many studies [10] and there is no consensus on the relationship between FBHC and pancreatic disease $[8,9]$.

We have found a significant increase in FBHC in patients with pancreatic duct stenosis, which suggests that a decline in exocrine pancreatic secretion induces changes in intestinal bacterial flora [11]. The objective of this study was to clarify the relationships among exocrine pancreatic secretion, FBHC and intestinal bacterial flora, and to investigate the usefulness of measuring FBHC as a simple diagnostic method for PEI.

\section{Methods}

\section{Subjects}

The subjects were patients aged $\geq 20$ years old who agreed to participate in the study. The subjects were prospectively collected from April 2019 to June 2020. All subjects were inpatients and were classified into those with $\mathrm{PC}, \mathrm{CP}$, other pancreatic diseases, and a normal pancreas. PC cases were histopathologically diagnosed with pancreatic duct cancer by surgery or EUS-guided fine needle aspiration. CP was diagnosed using the M-ANNHEIM criteria [12]. Patients admitted for a disease other than pancreatic disease and in whom pancreatic disease was excluded based on imaging and blood tests were included as subjects with a normal pancreas. The exclusion criteria were pregnancy; patients being fasted long term; use of antibiotics, probiotics, or pancreatic enzyme replacement drugs within one month before the test: history of surgery on the digestive tract or lung; presence of concomitant disease of cancer of other organs, stage 2 or advanced chronic renal failure, decompensated cirrhosis, active pulmonary disease, gastrointestinal obstruction, apparent gastrointestinal hemorrhage, caries being treated, or periodontal disease; no written consent, and judgement as inappropriate by a physician in charge $[8,9]$.

\section{Pancreatic function test}

The BT-PABA test and the 24-h urinary $\mathrm{C}$ peptide excretion (CPR) test were performed as exocrine and endocrine pancreatic secretion tests, respectively. These tests were performed under non-fasting conditions within one week before and after measuring breath hydrogen. In both tests, the measurement was repeated 3 times on different days and the mean was used for analysis [13]. A PABA excretion rate of $<73.4 \%$ was regarded as reduced exocrine pancreatic secretion [14], and a CPR rate of $<29.2 \mu \mathrm{g} /$ day was regarded as reduced endocrine pancreatic secretion.

\section{Breath sampling}

All patients ate a hospital meal on the day before the test and were fasted after 21:00 with drinking of water only. On the day of each breath test, the patients brushed their teeth at 7:00 a.m., breathed deeply twice, and held their breath for $15 \mathrm{~s}$ while end-expired breath was collected. Cigarette smoking, alcohol intake, excess exercise, and eating between meals were prohibited after admission. The collection method followed that of the Rome Consensus Conference and North American Consensus in 2017 [8, 9].

\section{Expired gas analysis}

A sensor gas chromatograph (SGHA, Nissha FIS Inc.) was used for measurements. Analysis of the results was performed using specialized SGC Analysis Software. Unlike general GC analysis, quantitation was performed using the peak height (= signal intensity). Hydrogen, carbon monoxide, and methane were measured and the target measurement level was 1.0-100 ppm.

\section{Sample collection of microbiota and 16S rRNA gene sequencing}

Feces collected during hospitalization were rapidly frozen. DNA was isolated from feces using a DNeasy PowerSoil Kit (Qiagen, Hilden, Germany) and amplified by targeting the V3-4 region of bacterial 16S rRNA using universal primers (forward: 5'-TCG TCG GCA GCG TCA GAT GTG TAT AAG AGA CAG CCT ACG GGN GGC WGC AG-3' and reverse: $5^{\prime}$-GTC TCG TGG GCT CGG AGA TGT GTA TAA GAG ACA GGA CTA CHV GGG TAT CTA ATC C-3'). The PCR products were pooled, and sequencing libraries were constructed and sequenced using an Illumina MiSeq sequencer. Pair-End Reads were prepared using MiSeq Reagent Kit v3 with $2 \times 300$ reads and 600 cycles (Illumina, San Diego, CA, USA). Analysis of $16 \mathrm{~S}$ rRNA gene sequence 
data was performed using USEARCH 6.1, Microbial Ecology (QIIME 1.9.1) and Greengenes v.13_8.

\section{Analytical methods}

For between-group comparison based on the BT-PABA test, the subjects were classified into PEI and non-PEI groups based on the criterion of a PABA excretion rate of $73.4 \%$. Associations of the PABA excretion rate were examined with age, height, body mass index (BMI), pancreatic disease ( $\mathrm{PC}, \mathrm{CP}$, and other pancreatic diseases), rates of concomitant diseases (hypertension, dyslipidemia, diabetes), history of alcohol intake, blood test findings (hemoglobin, HbA1c, creatinine, urea nitrogen, amylase, lipase, total protein, albumin, CEA, CA19-9), and presence of characteristic imaging findings in pancreatic disease (pancreatic hypertrophy, calcification, pancreatic cyst, main pancreatic duct (MPD) stenosis, and MPD dilatation). Concomitant diseases were defined as follows: hypertension, $\geq 140 / 90 \mathrm{mmHg}$ blood pressure or treatment with an oral hypotensive drug; dyslipidemia, $\geq 160 \mathrm{mg} / \mathrm{dL}$ LDL cholesterol or treatment with an LDL-lowering drug; and diabetes, $\geq 6.5 \%$ or higher hemoglobin A1c or under treatment $[15,16]$. Regarding alcohol ingestion, a subject with a history of continuous ingestion of $\geq 80 \mathrm{~g}$ pure ethanol a day [17] was defined as a heavy drinker. Pancreatic hypertrophy was defined using the criteria of Haage et al., in which the thicknesses of the pancreatic head and tail correspond to one or more vertebral bodies and $2 / 3$ or more of the vertebral body, respectively [18]. A MPD with a diameter $>3 \mathrm{~mm}$ was regarded as MPD dilatation. MPD stenosis was diagnosed using endoscopic retrograde cholangiopancreatography (ERCP), magnetic resonance cholangio pancreatgraphy (MRCP), and endoscopic ultrasound (EUS), and calcification and a pancreatic cyst were diagnosed using computed tomography (CT) and EUS.

The relationship between endocrine pancreatic secretion and fasting expired gas levels was investigated based on the associations of FBHC, fasting breath carbon monoxide concentration (FBCC), and fasting breath methane concentration (FBMC) with pancreatic function. For exocrine pancreatic secretion, intestinal bacterial flora were compared between the PEI and non-PEI groups.

\section{Statistical analysis}

Statistical analysis was performed using SPSS v.27.0 (IBM Corp.). All tests were 2 -sided and $P<0.05$ was regarded as significant. Continuous variables were analyzed as the median and range. Comparison of data that were not normally distributed was with a non-parametric Mann-Whitney-U test. Differences in rates between two groups were examined by Fisher exact test. Correlations between expired gas levels and pancreatic function tests were analyzed using a Spearman correlation coefficient (r). The cut-off value for FBHC for diagnosis of PEI was determined from a receiver operating characteristic (ROC) curve and the area under this curve (AUCROC), so as to maximize the Youden index (sensitivity + specificity-1). For between-group comparison of intestinal bacteria, LEfSe (http://hutte nhower.sph.harvard.edu/galaxy/) was used with default settings.

\section{Results}

\section{Patient background and clinical parameters}

Of 80 patients who were enrolled in the study between April 2019 and June 2020, the items described above could be analyzed in 60 . The excluded patients were those for whom feces could not be acquired during hospitalization $(\mathrm{n}=4)$, intestinal bacterial flora could not be analyzed due to the poor condition of fecal samples $(n=8)$, and antibiotic treatment was initiated before expired gas collection after admission $(n=8)$. The 60 patients were divided into PEI and non-PEI groups $(\mathrm{n}=30$ each) based on a PABA excretion rate of $73.4 \%$ (Table 1). The PEI group had a significantly higher rate of heavy drinkers $(P=0.023)$, a significantly lower level of serum albumin $(P=0.006)$, and a significantly higher rate of main pancreatic duct stenosis on imaging $(P=0.038)$. In contrast, the image findings of pancreas were more frequently normal in patients in the non-PEI group $(P=0.020)$.

\section{Expired gas analysis}

There was a strong positive correlation between FBHC and FBCC $(\mathrm{r}=0.754, P<0.001)$ and a negative correlation between FBHC and FBMC $(r=-0.387, P=0.001)$ (Fig. 1a, b). FBHC was significantly higher in the PEI group than in the non-PEI group (median: 2.80 (0.7$28.2)$ vs. 15.70 (1.4-77.0) ppm, $P<0.001$ ) (Fig. 2). Similarly, FBCC was significantly higher in the PEI group $(P=0.001)$, whereas there was no significant difference in FBMC $(P=0.216)$ (Table 2$)$. FBHC had a negative correlation with PABA excretion rate $(r=-0.523, P<0.001)$ and no correlation with the CPR value $(r=-0.067$, $P=0.608)$ (Fig. 3a, b). FBCC showed similar respective correlations $(\mathrm{r}=-0.462, P<0.001 ; \mathrm{r}=-0.091 P=0.487)$ since it was positively correlated with FBHC. FBMC was not correlated with the PABA excretion rate $(r=0.131$, $P=0.320)$ or $\mathrm{CPR}$ value $(r=0.081, P=0.541)$. An ROC curve for FBHC and PABA excretion rate was used to determine a cut-off value for FBHC (Fig. 4). A cut-off of $10.7 \mathrm{ppm}$ (AUCROC: $0.796,95 \%$ confidence interval: $0.678-0.913, P<0.001)$ gave a sensitivity of $73.3 \%$ and a specificity of $83.3 \%$ for diagnosis of PEI. 
Table 1 Patient background and clinical parameters

\begin{tabular}{|c|c|c|c|}
\hline & PEI group $(n=30)$ & Non-PEl group $(n=30)$ & $P$ value \\
\hline Age, median [range] & $71.0[46-87]$ & $71.0[40-81]$ & 1.000 \\
\hline Sex, male: female & $24: 6$ & $22: 8$ & 0.542 \\
\hline BMI, median [range] & 21.585 [15.99-28.07] & $21.090[15.24-26.38]$ & 0.255 \\
\hline History of alcohol intake, n (\%) & $19(63.3)$ & $19(63.3)$ & 1.000 \\
\hline Heavy drinkers, n (\%) & $7(23.3)$ & $1(3.3)$ & 0.023 \\
\hline Smoking history, n (\%) & $17(56.7)$ & $19(63.3)$ & 0.598 \\
\hline \multicolumn{4}{|l|}{ Pancreatic disease } \\
\hline$P C, n(\%)$ & $11(36.7)$ & $12(40.0)$ & 0.791 \\
\hline$C P, n(\%)$ & $8(26.7)$ & $3(10.0)$ & 0.095 \\
\hline Other pancreatic disease, n (\%) & $11(36.7)$ & $10(33.3)$ & 0.761 \\
\hline \multicolumn{4}{|l|}{ Concomitant diseases } \\
\hline Hypertension, n (\%) & $10(33.3)$ & $15(50.0)$ & 0.190 \\
\hline Dyslipidemia, n (\%) & $10(33.3)$ & $11(36.7)$ & 0.787 \\
\hline Diabetes, n (\%) & $14(46.7)$ & $13(43.3)$ & 0.795 \\
\hline \multicolumn{4}{|l|}{ Blood test findings } \\
\hline $\mathrm{Hb}(\mathrm{g} / \mathrm{dL})$ [range] & $13.50[7.9-15.5]$ & $13.50[10.9-15.9]$ & 0.877 \\
\hline BUN (mg/dL) [range] & $16.05[3.7-29.2]$ & $15.75[6.2-26.9]$ & 1.000 \\
\hline Cre (mg/dL) [range] & $0.775[0.49-1.32]$ & $0.730[0.42-1.24]$ & 0.225 \\
\hline $\mathrm{TP}(\mathrm{g} / \mathrm{dL})$ [range] & $7.00[5.5-8.0]$ & $7.20[6.2-7.8]$ & 0.716 \\
\hline Alb (g/dL) [range] & $3.95[1.4-4.5]$ & $4.20[3.6-4.7]$ & 0.006 \\
\hline Amy (IU/L) [range] & $83.5[30-374]$ & $74.5[36-350]$ & 0.877 \\
\hline Lipase (IU/L) [range] & $48.5[4-781]$ & 34.5 [10-889] & 0.706 \\
\hline HbA1c (NGSP \%) [range] & $6.65[4.9-13.7]$ & $6.20[4.9-10.5]$ & 0.195 \\
\hline CEA (ng/mL) [range] & $3.2[1.0-44.4]$ & $2.6[0.7-105.8]$ & 0.261 \\
\hline CA19-9 (U/mL) [range] & $119.50[<1-23210]$ & $61.50[<1-197000]$ & 0.446 \\
\hline \multicolumn{4}{|l|}{ Imaging findings } \\
\hline Pancreatic hypertrophy, n (\%) & $9(30.0)$ & $3(10.0)$ & 0.053 \\
\hline Calcification, n (\%) & $4(13.3)$ & $2(6.7)$ & 0.389 \\
\hline Pancreatic cyst, n (\%) & $6(20.0)$ & $5(16.7)$ & 0.739 \\
\hline MPD dilatation, n (\%) & $19(63.3)$ & $13(43.3)$ & 0.121 \\
\hline MPD stenosis, n (\%) & $20(66.7)$ & $12(40.0)$ & 0.038 \\
\hline Normal pancreas, n (\%) & $0(0.0)$ & $5(16.7)$ & 0.020 \\
\hline
\end{tabular}

$P E I$ pancreatic exocrine insufficiency, $B M I$ body mass index, $P C$ pancreatic carcinoma, $C P$ chronic pancreatitis, $H b$ hemoglobin, $B U N$ blood urea nitrogen, $C r e$ creatinine, TP total protein, Alb albumin, Amy amylase, HbA1c hemoglobin A1c, NGSP national glycohemoglobin standardization program, CEA carcinoembryonic antigen, CA19-9 carbohydrate antigen 19-9, MPD major pancreatic duct

\section{PABA excretion rate-associated changes in intestinal bacterial flora}

The bacteria proportions at the 'phylum' level in the PEI and non-PEI groups are shown in Fig. 5a. Bacteroidetes was dominant in both groups. The occupancy by the phylum Firmicutes was higher in the PEI group, and those for Proteobacteria, Verrucomicrobia, and Fusobacteria were lower. At the 'genus' level, the occupancies by the genera Blautia, Faecalibacterium, and Streptococcus were higher and those of Parabacteroides and Akkermansia were low in the PEI group (Fig. 5b). A comparison of microbiomes showed increases in the genera Clostridium, Lachnospira, Veillonella, Selenomonas, and Anaerococcus belonging to the class Clostridia, which are obligate anaerobes, in the PEI group $(P<0.05)$. Increases in the genera Enterococcus and Lactobacillus belonging to the family Lactobacillaceae, and genera Leptotrichia, Prevotella, Serratia, and Aggregatibacter belonging to the family Leptotrichiaceae were also noted (Fig. 5c) $(P<0.05)$.

\section{Discussion}

PERT can improve the quality of life and life expectancy of PEI patients [3, 4]. However, not all patients with pancreatic diseases are being evaluated for PEI because complicated tests are required for its diagnosis. PERT is also difficult for all patients because it needs much cost 

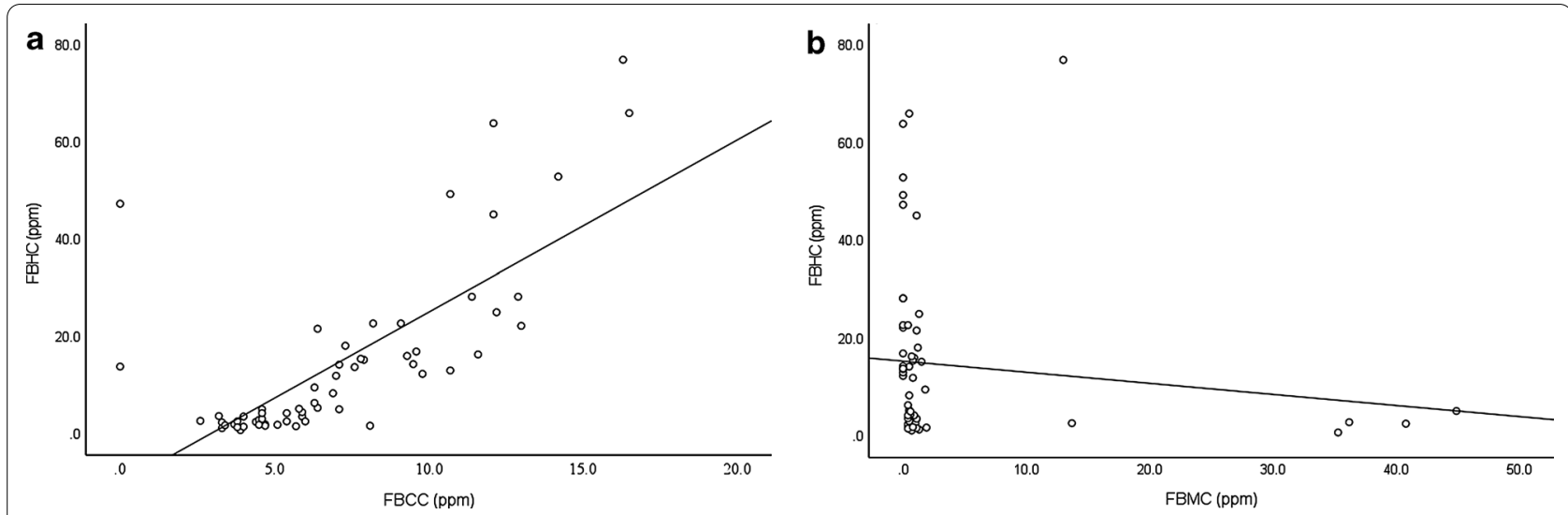

Fig. 1 Correlation of fasting breath hydrogen concentration (FBHC) with fasting breath carbon monoxide concentration (FBCC) and fasting breath methane concentration (FBMC). a Fasting breath hydrogen concentration (FBHC) and fasting breath carbon monoxide concentration (FBCC). There was a strong positive correlation between FBHC and FBCC $(r=0.754, P<0.001)$. b Fasting breath hydrogen concentration (FBHC) and fasting breath methane concentration (FBMC). There was a negative correlation between FBHC and FBMC $(r=-0.387, P=0.001)$

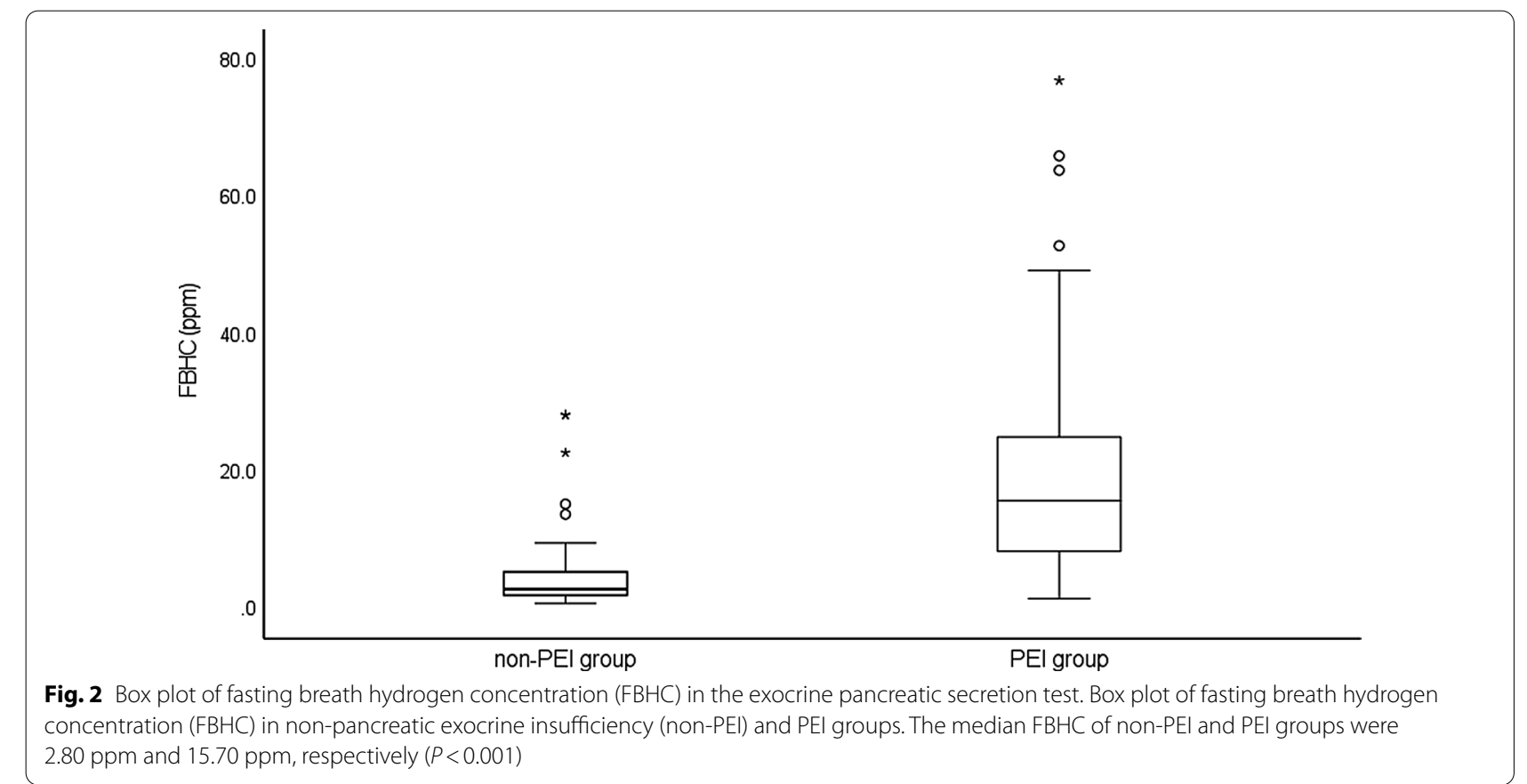

Table 2 Comparison of expired gas in PEl and non-PEl groups

\begin{tabular}{lrcr}
\hline & PEl group $(\mathbf{n}=\mathbf{3 0})$ & $\begin{array}{l}\text { Non-PEl group } \\
(\mathbf{n = 3 0})\end{array}$ & P value \\
\hline FBHC (ppm) [range] & $15.70[1.4-77.0]$ & $2.80[0.7-28.2]$ & $<0.001$ \\
FBCC (ppm) [range] & $8.15[0.0-16.5]$ & $4.90[2.6-12.9]$ & 0.001 \\
FBMC (ppm) [range] & $0.55[0.0-36.2]$ & $0.70[0.0-44.9]$ & 0.216
\end{tabular}

$P E I$ pancreatic exocrine insufficiency, $F B H C$ fasting breath hydrogen concentration, $F B C C$ fasting breath carbon monoxide concentration, $F B M C$ fasting breath methane concentration, ppm parts per million and many tablets. In order to provide PERT to appropriate patients, the development of a diagnosis of PEI that is simple and can be easily performed in an outpatient is required.

BHT has the potential to diagnose PEI. In humans, hydrogen is only produced in the intestine through degradation and metabolism of unabsorbed food by intestinal bacteria, and about $14 \%$ is excreted via expiration [19]. Since a decline in exocrine pancreatic secretion 



Fig. 3 Correlation between breath hydrogen concentration (FBHC) and pancreatic function. a The result of BT-PABA test. FBHC had a negative correlation with PABA excretion rate $(r=-0.523, P<0.001)$. $\mathbf{b}$ The result of 24 urinary $C$ peptide excretion (CPR) test. There was no significant correlation between FBHC and CPR $(r=-0.067, P=0.608)$

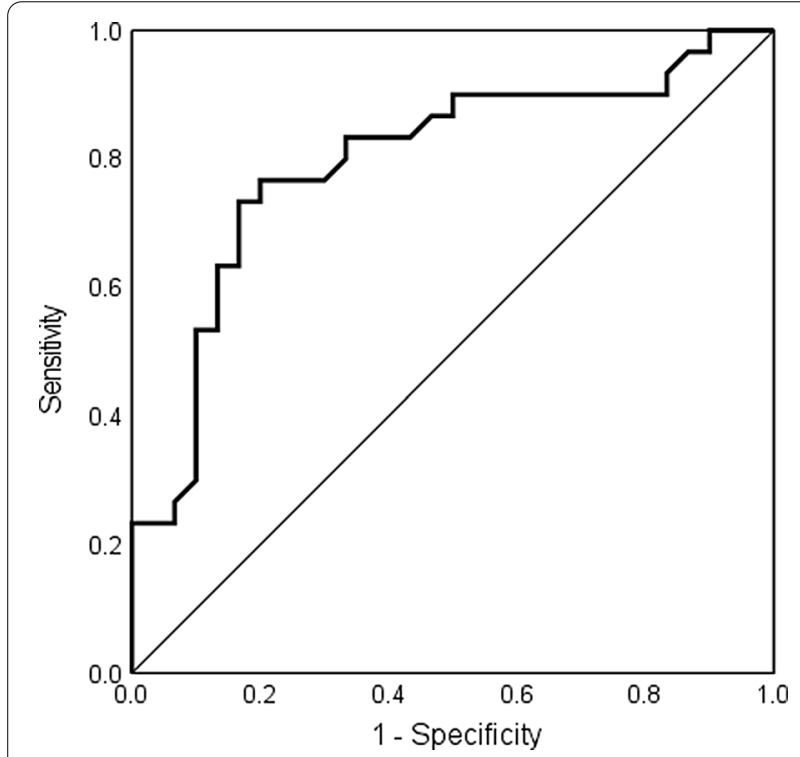

Fig. 4 Diagnostic accuracy of pancreatic exocrine insufficiency (PEI) due to elevated fasting breath hydrogen concentration (FBHC) levels. An receiver operator characteristic $(\mathrm{ROC})$ curve for fasting breath hydrogen concentration (FBHC) and PABA excretion rate. The cut-off value for the highest sensitivity with maintaining specificity was 10.7 ppm for diagnosis of PEI (the area under ROC (AUCROC): 0.796, 95\% confidence interval: $0.678-0.913, P<0.001)$

is known to induce changes in intestinal bacterial flora, this study was performed with the hypothesis that simple FBHC measurement may be useful to predict PEI.

In this study, FBHC was significantly higher in the PEI group and had a negative correlation with the PABA excretion rate. Thus, the breath hydrogen concentration increased as exocrine pancreatic secretion decreased. The normal limit of FBHC is unclear, but this value varies from 10 to $20 \mathrm{ppm}$ [20]. At present, $\geq 20 \mathrm{ppm}$, the diagnostic criterion for SIBO, is the accepted value $[8,9,20]$, but $F B H C \geq 20$ ppm was found in only 12 of 30 patients in the PEI group (and in none in the nonPEI group). This suggests that $\geq 20 \mathrm{ppm}$ is inappropriate as a screening criterion because it may increase the false negative rate. A cut-off value of FBHC of $10.7 \mathrm{ppm}$ gave favorable sensitivity and specificity for diagnosis of PEI in this study.

Regarding other expired gas, cross-sensitivity of FBCC with the hydrogen concentration has been shown [21] and a strong positive correlation with FBHC was found in the current study. FBHC has been found to decrease in subjects with high FBMC, and these variables had an inverse correlation in this study. Fewer of our subjects produced methane compared with the rate of $44 \%$ in a previous study, and we did not find an association of FBMC with pancreatic function [22].

To elucidate this mechanism, we also focused on the relationship between FBHC and microbiome. In comparison of intestinal bacterial flora, as previously reported, increases in the division Firmicutes [23, 24], genus Clostridium [25], and family Lactobacillaceae [26] were observed in PC, CP, and PEI. Of 343 species of intestinal bacteria registered in the Human Microbiome Project, about $71 \%$ encode hydrogenase, an enzyme catalyzing a reversible oxidation-reduction reaction of hydrogen. The division Firmicutes, which significantly increased in the PEI group, accounts for $21 \%$ of the hydrogenase content in bacterial flora in the human colon [27]. The genus Clostridium is a representative hydrogen-producing bacteria producing $1.1-2.3 \mathrm{~mol} \mathrm{H}_{2} / \mathrm{mol}$ glucose at temperatures $\left(30-40{ }^{\circ} \mathrm{C}\right)$ close to that of the human body and has been widely studied in the biomass field $[28,29]$. 


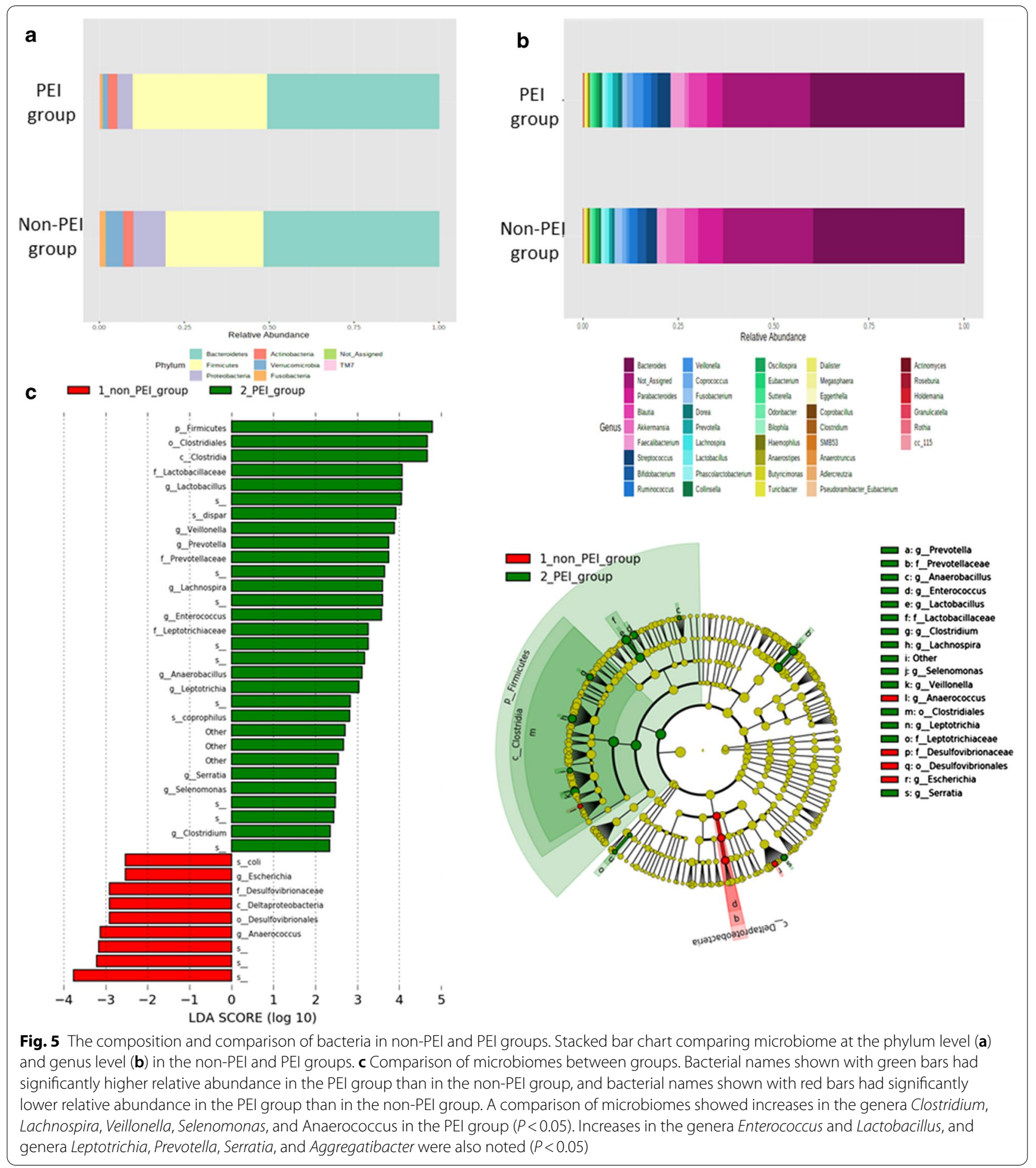

The genus Clostridium is one of the main hydrogen sources in the human intestine [30] and is likely to have been the cause of elevation of FBHC in the PEI group. In addition, some of Clostridium spp. form a carbohydrateassociated enzyme complex (a "cellulosome") and 57 cellulosome genes encoding lipase, peptidase, and proteinase inhibitors, in addition to carbohydrate-activating enzymes, have been discovered [31]. Since undigested food flows in the intestine due to PEI, Clostridium with these characteristics may have increased and resulted 
in an increase in hydrogen. The genus Lactobacillus of the family Lactobacillaceae also produces hydrogen in a medium temperature environment [32], and a state with reduced exocrine secretion (i.e., an intestinal condition with undigested food) may be an advantageous environment for a hydrogen-producing anaerobe.

PERT has been shown to improve undernutrition, gastrointestinal symptoms, and QOL by decreasing undigested food [4]. This suggests that an increase in undigested food is reflected as FBHC elevation, suggesting its potential as an effective pre-test marker before use of PERT. A bag is the only running cost required for breath hydrogen measurement, which reduces the cost. The test time is about $1 \mathrm{~min}$ and the measurement time is about $4 \mathrm{~min}$, which are shorter than those in other tests, and the test is non-invasive and can be performed repeatedly. Thus, it may also be useful for judgment of the effect of PERT, as well as for screening prior to treatment.

Several diagnoses of PEI have been used clinically. Faecal elastase-1 (FE-1) has been used as a reliable diagnostic method for PEI, but a faecal sample is required [33]. Faecal collection can be difficult for outpatients, especially for the elderly patients [34]. In addition, the sensitivity of FE-1 has been reported to be low in mild to moderate PEI patients [33]. The 13C-Mixed Triglyceride Breath Test (13C-MTBT) has been reported to be potentially useful [35]. However, there is not enough evidence for clinical application, and it requires long examination time and fasting. We compared FBHC with BT-PABA as a control, but could not compare it with other tests because of the limitation of public insurance in Japan. However, we believe that FBHC is cheaper and easier than these methods, and is useful for early diagnosis of PEI.

There are several limitations in this study. First, all subjects were inpatients with diseases, and no samples were obtained from healthy persons. Therefore, we could not eliminate the confounding factors such as alcohol consumption, smoking, and diabetes. that may have affected the results of the BHT and microbiome analysis [36, 37]. Second, there were only a few patients with FBMC elevation and the clinical significance of this effect could not be analyzed. Production of methane consumes hydrogen and this may latently influence hydrogen measurement [22]. Third, we could not clarify the relationship with the concept of SIBO because we did not use glucose or lactose loading for BHT. We used the average value of three times to increase the reliability of the score, but the accuracy of FBHC for PEI diagnosis did not meet our expectations at the present setting. It is necessary to increase the number of patients including healthy controls and measure both fasting and glucose loaded hydrogen breath levels to solve these problems.

\section{Conclusion}

Patients with PEI have elevated FBHC levels, which may be related to the genus Clostridium producing hydrogen in the gut. FBHC measurement may be useful in the diagnosis of PEI.

\begin{abstract}
Abbreviations
PEl: Pancreatic exocrine insufficiency; FBHC: Fasting breath hydrogen concentration; Cl: Confidence interval; QOL: Quality of life; PERT: Pancreatic enzyme replacement therapy; PC: Pancreatic carcinoma; CP: Chronic pancreatitis; BHT: Breath hydrogen test; SIBO: Small intestinal bacterial overgrowth syndrome; CPR: The 24-h urinary C peptide excretion test; BMI: Body mass index; MPD: Main pancreatic duct; ERCP: Endoscopic retrograde cholangiopancreatography; MRCP: Magnetic resonance cholangiopancreatgraphy; EUS: Endoscopic ultrasound; CT: Computed tomography; FBCC: Fasting breath carbon monoxide concentration; FBMC: Fasting breath methane concentration; ROC: Receiver operating characteristic.
\end{abstract}

\section{Acknowledgements}

We thank Ms. Akina Ooishi of the Department of Gastroenterology and Hepatology, Nagoya University Graduate School of Medicine for technical assistance in DNA isolation and 16S rRNA gene sequencing.

\section{Authors' contributions}

Study concept and design: KU, KY, HK, TH, MN, MI, OE, TI 1, TI 2. Analysis and interpretation of the data: YK, KU, HK. Drafting of the article: KU, KM, HK, MF. All authors read and approved the final manuscript.

\section{Funding}

No funding was obtained for this study.

\section{Availability of data and materials}

The data of this study are available from the corresponding author upon reasonable request.

\section{Declarations}

\section{Ethics approval and consent to participate}

This study was performed after approval by the Ethics Review Committee of Nagoya University Hospital and with written consent from the subjects after they received an explanation of the study (UMIN000020269, UMIN000020777). The study followed the ethics criteria established in the Declaration of Helsinki and subsequent revisions. All study participants provided informed written consent prior to be enrolled in this study

Consent for publication

Not applicable.

\section{Competing interests}

The authors declare that they have no competing interests.

\section{Author details}

${ }^{1}$ Department of Gastroenterology and Hepatology, Nagoya University Graduate School of Medicine, 65 Tsuruma-cho, Showa-ku, Nagoya 466-8550, Japan. ${ }^{2}$ Department of Endoscopy, Nagoya University Hospital, 65 Tsuruma-cho, Showa-ku, Nagoya 466-8550, Japan. ${ }^{3}$ Department of Gastroenterology and Gastroenterological Oncology, Fujita Health University, 1-98 Dengakugakubo, Kutsukake-cho, Toyoake, Aichi 470-1192, Japan.

Received: 29 January 2021 Accepted: 20 April 2021

Published online: 10 May 2021

\footnotetext{
References

1. Lindkvist B. Diagnosis and treatment of pancreatic exocrine insufficiency. World J Gastroenterol. 2013;19:7258-66.
} 
2. Capurso G, Traini M, Piciucchi M, et al. Exocrine pancreatic insufficiency: prevalence, diagnosis, and management. Clin Exp Gastroenterol. 2019:12:129-39.

3. Bartel MJ, Asbun H, Stauffer J, et al. Pancreatic exocrine insufficiency in pancreatic cancer: a review of the literature. Dig Liver Dis. 2015;47:1013-20.

4. de la Iglesia-García D, Huang W, Szatmary P, et al. Efficacy of pancreatic enzyme replacement therapy in chronic pancreatitis: systematic review and meta-analysis. Gut. 2017:66:1354-5.

5. Carnie LE, Lamarca A, McNamara MG, et al. The assessment of pancreatic exocrine function in patients with inoperable pancreatic cancer: in need of a new gold-standard. Pancreatology. 2020;20:668-75.

6. Lee AA, Baker JR, Wamsteker EJ, et al. Small intestinal bacterial overgrowth is common in chronic pancreatitis and associates with diabetes, chronic pancreatitis severity, low zinc levels, and opiate use. Am J Gastroenterol. 2019;114:1163-71.

7. Ní Chonchubhair HM, Bashir Y, Dobson M, et al. The prevalence of small intestinal bacterial overgrowth in non-surgical patients with chronic pancreatitis and pancreatic exocrine insufficiency (PEI). Pancreatology. 2018;18:379-85.

8. Ali R, Michelle B, Anthony L, et al. Hydrogen and methane-based breath testing in gastrointestinal disorders: the North American consensus. Am J Gastroenterol. 2017;112:775-84

9. Gasbarrini A, Corazza GR, Gasbarrini G, et al. Methodology and indications of H2-breath testing in gastrointestinal diseases: The Rome Consensus Conference. Aliment Pharmacol Ther. 2009;29(Suppl 1):1-14.

10. Avallone EV, De Carolis A, Loizos P, et al. Hydrogen breath test: diet and basal H2 excretion: a technical note. Digestion. 2010;82:39-41.

11. Sakai D, Hirooka Y, Kawashima $H$, et al. Increase in breath hydrogen concentration was correlated with the main pancreatic duct stenosis. J Breath Res. 2018:12:036004. https://doi.org/10.1088/1752-7163/aaaf77.

12. Schneider AJ, Lohr M, Singer MV. The M-ANNHEIM classification of chronic pancreatitis: introduction of a unifying classification system based on a review of previous classifications of the disease. J Gastroenterol. 2007;42:101-19.

13. Hayashi D, Hirooka Y, Kawashima $H$, et al. Functional cor relation between the pancreas and the small intestine in humans. Pancreas. 2018;47:601-8.

14. Yamato C, Kinoshita K. A simple assay for measurement of urinary p-aminobenzoic acid in the oral pancreatic function test. Anal Biochem. 1979:98:13-7.

15. Chamberlain JJ, Rhinehart AS, Shaefer CF, et al. A diagnosis and management of diabetes: synopsis of the 2016 American Diabetes Association Standards of Medical Care in Diabetes. Ann Intern Med. 2016;164:542-52.

16. Expert panel on detection, evaluation treatment of high blood cholesterol in adults. Executive summary of the Third Report of The National Cholesterol Education Program (NCEP) Expert Panel on Detection, Evaluation, And Treatment of High Blood Cholesterol In Adults (Adult Treatment Panel III). JAMA. 2001;285:2486-2497.

17. Ito $\mathrm{T}$, Ishiguro $\mathrm{H}$, Ohara $\mathrm{H}$, et al. Evidence-based clinical practice guidelines for chronic pancreatitis 2015. J Gastroenterol. 2016;51:85-92.

18. Haaga JA, Alfidi RJ, Zelch MG, et al. Computed tomography of the pancreas. Radiology. 1976;120:589-95.

19. Levitt MD. Production and excretion of hydrogen gas in man. N Engl J Med. 1969;281:122-7.

20. Signoretti M, Stigliano S, Valente R, et al. Small intestinal bacterial overgrowth in patients with chronic pancreatitis. J Clin Gastroenterol. 2014;48(Supp 1):S52-5.

21. Costello BP, Ewen RJ, Ratcliffe NM. A sensor system for monitoring the simple gases hydrogen, carbon monoxide, hydrogen sulfide, ammonia and ethanol in exhaled breath. J Breath Res. 2008;2:037011. https://doi. org/10.1088/1752-7155/2/3/037011.

22. de Lacy Costello BP, Ledochowski M, Ratcliffe NM. The importance of methane breath testing: a review. J Breath Res. 2013;7:024001. https://doi. org/10.1088/1752-7155/7/2/024001.

23. Jandhyala SM, Madhulika A, Deepika G, et al. Altered intestinal microbiota in patients with chronic pancreatitis: implications in diabetes and metabolic abnormalities. Sci Rep. 2017;7:43640. https://doi.org/10.1038/srep4 3640.

24. Ren Z, Jiang J, Xie H, et al. Gut microbial profile analysis by MiSeq sequencing of pancreatic carcinoma patients in China. Oncotarget. 2017:8:95176-91.

25. Hamada S, Masamune A, Nabeshima T, et al. Differences in gut microbiota profiles between autoimmune pancreatitis and chronic pancreatitis. Tohoku J Exp Med. 2018;244:113-7.

26. Isaiah A, Parambeth JC, Steiner JM, et al. The fecal microbiome of dogs with exocrine pancreatic insufficiency. Anaerobe. 2017;45:50-8.

27. Wolf PG, Biswas A, Morales SE, et al. $\mathrm{H} 2$ metabolism is widespread and diverse among human colonic microbes. Gut Microbes. 2016;7:235-45.

28. Latifi A, Avilan L, Brugna M. Clostridial whole cell and enzyme systems for hydrogen production: current state and perspectives. Appl Microbiol Biotechnol. 2019;103:567-75.

29. Hendrickx M, De Vos P, De Ley J. Fermentation characteristics of clostridium pasteurianum LMG3285 grown on glucose and mannitol. J Appl Bacteriol. 1991;70:52-8.

30. Suzuki A, Ito M, Hamaguchi T, et al. Quantification of hydrogen production by intestinal bacteria that are specifically dysregulated in Parkinson's disease. PLoS ONE. 2018;13:e0208313. https://doi.org/10.1371/journal. pone.0208313.

31. Tamaru Y, Miyake H, Kuroda K, et al. Comparative genomics of the mesophilic cellulosome-producing Clostridium cellulovorans and its application to biofuel production via consolidated bioprocessing. Environ Technol. 2010;31:889-903.

32. Kim DH, Han SK, Kim SH, et al. Effect of gas sparging on continuous fermentative hydrogen production. Int J Hydrog Energy. 2006;3:2158-69.

33. Vanga RR, Tansel A, Sidiq S, et al. Diagnostic performance of measurement of fecal elastase-1 in detection of exocrine pancreatic insufficiency: systematic review and meta-analysis. Clin Gastroenterol Hepatol. 2018;16(8):1220-1228.e4.

34. McCrea GL, Miaskowski C, Stotts NA, et al. A review of the literature on gender and age differences in the prevalence and characteristics of constipation in North America. J Pain Symptom Manag. 2009;37(4):737-45.

35. Domínguez-Muñoz JE, Nieto L, Vilariño M, et al. Development and diagnostic accuracy of a breath test for pancreatic exocrine insufficiency in chronic pancreatitis. Pancreas. 2016;45(2):241-7.

36. Sasaki M, Ogasawara N, Funaki Y, et al. Transglucosidase improves the gut microbiota profile of type 2 diabetes mellitus patients: a randomized double-blind, placebo-controlled study. BMC Gastroenterol. 2013;13:81.

37. Bode C, Bode JC. Activation of the innate immune system and alcoholic liver disease: Effects of ethanol per se or enhanced intestinal translocation of bacterial toxins induced by ethanol? Alcohol Clin Exp Res. 2005;29:166S-S171.

\section{Publisher's Note}

Springer Nature remains neutral with regard to jurisdictional claims in published maps and institutional affiliations. 